\title{
Signal change in hippocampus and current source of spikes in Panayiotopoulos syndrome
}

Jung Sook Yeom, $\mathrm{MD}^{1,2}$, Youngsoo Kim, MD, $\mathrm{PhD}^{3}$, Ji Sook Park, $\mathrm{MD}^{1,2}$, Ji Hyun Seo, MD, $\mathrm{PhD}^{1,2}$, Eun Sil Park, MD, PhD ${ }^{1,2}$, Jae Young Lim, MD, PhD ${ }^{1,2}$, Chan-Hoo Park, MD, PhD ${ }^{1,2}$, Hyang Ok Woo, MD, PhD ${ }^{1,2}$, Hee-Shang Youn, $\mathrm{MD}, \mathrm{PhD}^{1,2}$, Oh-Young Kwon, MD, PhD ${ }^{2,3}$

${ }^{1}$ Department of Pediatrics, ${ }^{2}$ Gyeonsang Institute of Health Science, ${ }^{3}$ Department of Neurology, Gyeongsang National University School of Medicine, Jinju, Korea

Received: 17 May 2011, Revised: 21 June 2011 Accepted: 12 August 2011

Corresponding author: Oh-Young Kwon, MD, PhD Department of Neurology, Gyeongsang National University Hospital, Gyeongsang National University School of Medicine, 79 Gangnam-ro, Jinju 660-702, Korea

Tel: +82-55-750-8077, Fax: +82-55-755-1709

E-mail:mnkwon21@daum.net

Copyright $(C 2012$ by The Korean Pediatric Society
A 4-year-old girl with Panayiotopoulos syndrome presented with a history of 4 prolonged autonomic seizures. The clinical features of her seizures included, in order of occurrence, blank staring, pallor, vomiting, hemi-clonic movement on the right side, and unresponsiveness. A brain magnetic resonance imaging (MRI) showed a slightly high $\mathrm{T}_{2}$ signal in the left hippocampus. Interictal electoencephalogram revealed spikes in the occipital area of the left hemisphere. We analyzed the current-source distribution of the spikes to examine the relationship between the current source and the high $T_{2}$ signal. The current source of the occipital spikes was not only distributed in the occipital area of both cerebral hemispheres, but also extended to the posterior temporal area of the left hemisphere. These findings suggest that the left temporal lobe may be one of the hyperexcitable areas and form part of the epileptogenic area in this patient. We hypothesized that the high $\mathrm{T}_{2}$ signal in the left hippocampus of our patient may not have been an incidental lesion, but instead may be related to the underlying electroclinical diagnosis of Panayiotopoulos syndrome, and particularly seizure. This notion is important because an abnormal $T_{2}$ signal in the hippocampus may represent an acute stage of hippocampal injury, although there is no previous report of hippocampal pathology in Panayiotopoulos syndrome. Therefore, long-term observation and serial follow-up MRIs may be needed to confirm the clinical significance of the $\mathrm{T}_{2}$ signal change in the hippocampus of this patient.

Key words: Electroencephalography, Hippocampus, Childhood benign focal epilepsy

This is an open-access article distributed under the terms of the Creative Commons Attribution Non-Commercial License (http://creativecommons.org/licenses/by$\mathrm{nc} / 3.0 /$ ) which permits unrestricted non-commercial use, distribution, and reproduction in any medium, provided the original work is properly cited.

\section{Introduction}

Panayiotopoulos syndrome (PS) is the second most common benign idiopathic childhood epilepsy syndrome after Rolandic epilepsy ${ }^{1)}$. This syndrome is defined as 'a benign age-related focal seizure disorder occurring in early and mid-childhood; it is characterized by seizures, often prolonged, with predominantly autonomic symptoms, and by an electroencephalogram (EEG) that shows shifting and/or multiple foci, often with occipital predominace ${ }^{22}$. PS occurs in children who are otherwise normal and it is not associated with significant neurodevelopmental problems ${ }^{3}$. Although high resolution brain magnetic resonance imaging (MRI) is normal in PS, incidental brain lesions 
have been reported in this syndrome $e^{4)}$. However, hippocampal pathology has not been reported in PS.

We observed a slightly high $\mathrm{T}_{2}$ signal in the left hippocampus and an EEG with left occipital spikes in a 4-year-old girl with a typical clinical presentation of PS. The hippocampal $\mathrm{T}_{2}$ signal changes in this case could be interpreted in several ways, but we hypothesized that the changes were related to an underlying electroclinical diagnosis of PS, particularly seizure. Seizure-induced transient focal abnormalities at various cerebral regions, including the hippocampus, in $\mathrm{T}_{2}$-weighted MRI that may reflect transient vasogenic or cytogenic edema at the seizure focus have been reported ${ }^{5,6}$. This notion is important because an abnormal $T_{2}$ signal in the hippocampus may represent an acute stage of hippocampal injury that will progress to hippocampal sclerosis ${ }^{7}$.

We were unable to record ictal EEG signals in this patient; thus, we performed a current-source analysis of the interictal spikes and investigated the connection between the results and the high $\mathrm{T}_{2}$ signal based on the relationship between the irritative zone (area of cortex that generates interictal spikes) and ictal onset zone (area of cortex that initiates or generates seizures) ${ }^{8)}$.

\section{Case report}

A 4-year old girl suffered four prolonged autonomic seizures. She had not experienced any initial precipitating injury such as perinatal problems, head trauma, central nervous system infection, febrile convulsion, etc. The first seizure occurred 1 year before the fourth seizure. The fourth seizure occurred half an hour after falling asleep; she awoke suddenly, stared blankly, looked pale, and then vomited for 10 minutes. She could not respond to verbal commands during the late stage of vomiting. Afterward, her eyes deviated to the right side and clonic movements of the right arm followed. The hemi-clonic seizures lasted 10 to 15 minutes. After being unresponsive and flaccid for half an hour in the emergency room, she gradually recovered and felt normal. No problems in her neurological examination were found after her recovery. However, arterial blood gas analysis during unresponsiveness in emergency room showed respiratory acidosis with hypoxemia with followings; $\mathrm{pH}$ 7.14, $\mathrm{pCO}_{2} 88$ mmHg, $\mathrm{pO}_{2} 50$ $\mathrm{mmHg}$, bicarbonate $29 \mathrm{mmol} / \mathrm{L}$.

The EEG revealed repetitive bursts of sequential spikes and waves at the left occipital electrode (O1) (Fig. 1). The diagnosis of PS was made based on the ictal symptoms and the EEG findings. The brain MRI showed a slightly increased signal change in the left hippocampus on $\mathrm{T}_{2}$ and fluid-attenuated inversion recovery image (Fig. 2). Oxcarbazepine monotherapy was started, and she has been seizure free for 16 months on the monotherapy.

\section{Current source analysis of interictal spikes}

The EEG was recorded for at least 30 minutes using a 32-channel digital EEG machine (Comet EEG machines, Grass Technologies, West Warwick, RI, USA). We used 25 electrodes (Fp1/Fp2, F7/F8, F9/F10, T7/T8, T9/T10, P7/P8, P9/P10, F3/F4, C3/C4, P3/P4, O1/ $\mathrm{O} 2, \mathrm{Fz}, \mathrm{Cz}, \mathrm{Pz}$ ) of 10/20 International System to record scalp EEG signal. The sampling rate was $400 \mathrm{~Hz}$, and the bandwidth was set at 1 to $70 \mathrm{~Hz}$.

Forty 1-second epochs, including the 500-ms periods before and after the maximum negativity of the spikes, were selected from the interictal spikes at the $\mathrm{O} 1$ electrode. We averaged the selected 40 epochs into a single spike with brain electrical source analysis software ver. 5.3.6 (MEGIS, Gräfelfing, Germany).

To evaluate the cortical distribution of the current source of the averaged spikes, low resolution brain electromagnetic tomography (LORETA) software (KEY Institute for Brain-Mind Research, Zurich,

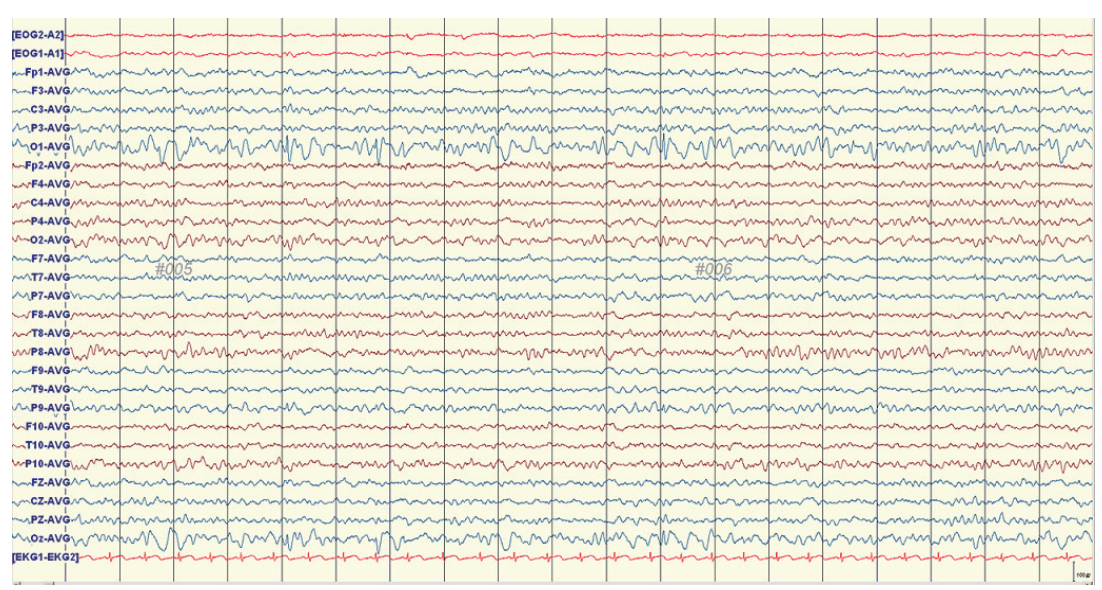

Fig. 1. An electroencephalogram taken just after the patient's fourth prolonged autonomic seizure. Spike-and-wave patterns of high amplitude are at the left occipital electrode with normal background activity. 
Switzerland) was used as a distributed model for the current source analysis. LORETA calculates and images the three-dimensional currentdensity distribution by assuming that the smoothest of all possible inverse solutions is the most plausible and using a three-shell spherical head model registered to the Talairach human brain atlas (McGill University, Montreal Neurologic Institute, McConnell Brain Imaging Center, Montreal, Canada). A current density over the 95th percentile was considered the threshold of significance. The current source of the occipital spikes was not only distributed in the occipital area of both cerebral hemispheres but also extended to the posterior temporal area of the left hemisphere (Fig. 3).

\section{Discussion}

Panayiotopoulos syndrome is a benign idiopathic epilepsy with interictal EEG features of repetitive occipital spikes". However, the concept of occipital lobe epilepsy can be difficult to explain due to the important ictal manifestations of autonomic symptoms and long

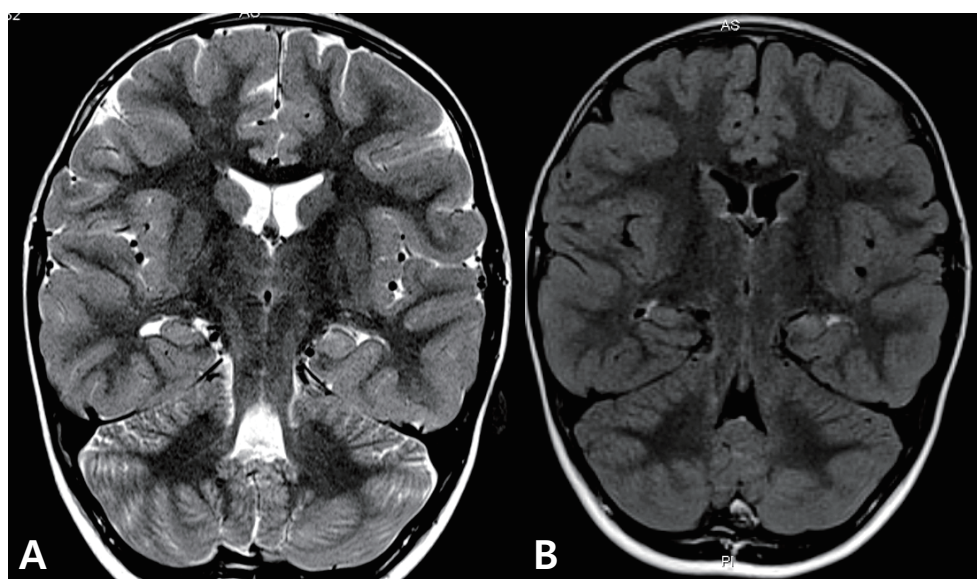

Fig. 2. Coronal sections of brain magnetic resonance imagings taken just after the patient's fourth prolonged autonomic seizure. Slightly increased signal intensity on the $\mathrm{T}_{2}(\mathrm{~A})$ and fluid-attenuated inversion recovery image (B) were observed. Structures of the left hippocampus are less obvious than the right.

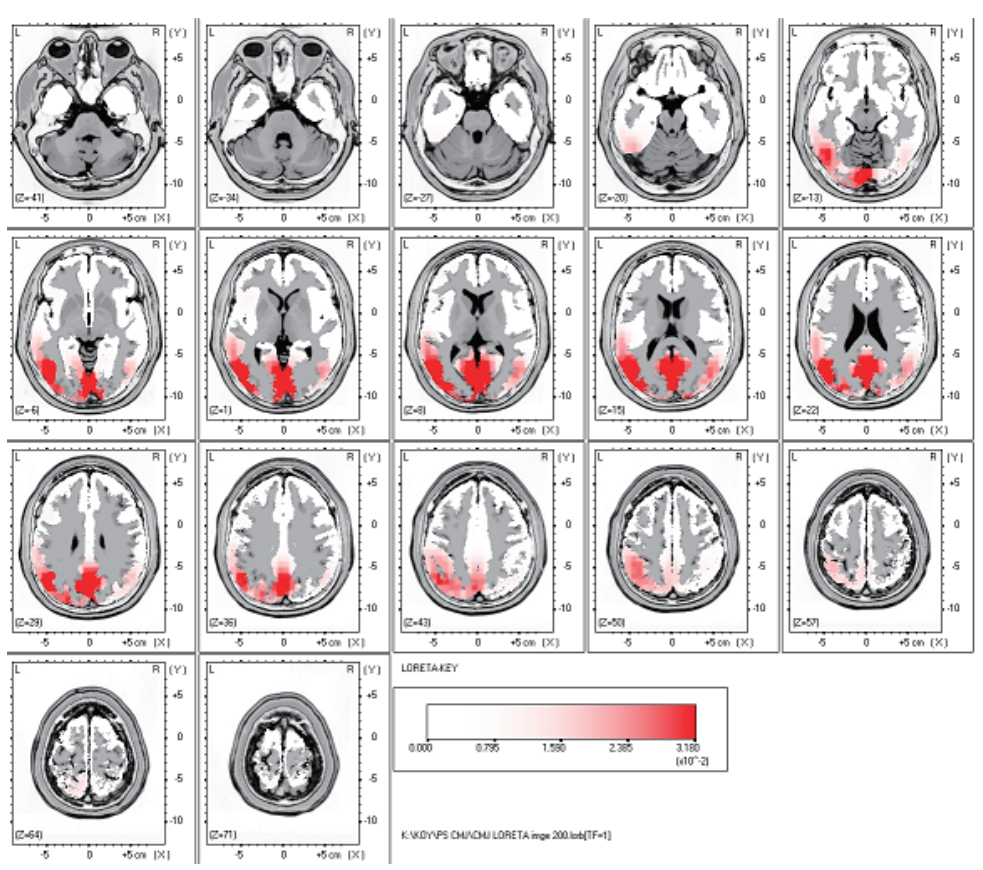

Fig. 3. Results of the current source analysis of the interictal spikes on the left occipital area in the 4-year-old girl with Panayiotopoulos syndrome. The current source at the negative peak of the averaged spike was not only distributed in the occipital area of both cerebral hemispheres, but also extended to the posterior temporal area of the left hemisphere. The letters $L$ and $R$ stand for left and right side. 
periods of interrupted consciousness, with rare visual symptoms. In addition, groups of autonomic seizures with extraoccipital foci or normal EEG were defined ${ }^{2}$. Subsequently, the concept of PS has been changed, and the understanding of PS has been expanded to include age-related multifocal cortical hyperexcitability and an unstable autonomic system ${ }^{2)}$.

Attempts to investigate the particular cortical generators of the interictal spikes also contributed to the conceptual changes in PS. In recent studies, using current source analysis of EEG, simultaneous EEG/ functional MRI, and magnetoencephalography, it was found that the epileptic activity of PS originates in the lateral occipital cortex and rapidly spreads to the cortical areas of the temporal or parietal lobes ${ }^{10,11)}$ or the temporocentrofrontal cortex ${ }^{12)}$. In another study that reviewed ictal EEGs revealed that there was no specific focus related to the autonomic symptoms. They suggested that autonomic symptoms of PS are likely to be generated by variable and widely spread epileptogenic foci acting on a temporarily hyperexcitable central autonomic network ${ }^{13}$. The temporal area may also be included in the network. The studies about interictal and ictal discharges of PS suggest that the temporal lobe may be one of the hyperexcitable or irritative areas and part of the epileptogenic area in PS patients ${ }^{11-13)}$.

The results of our case study are consistent with the current concept of PS. In our case, the interictal spikes were observed in the left occipital area. The current-source distribution of the interictal spikes was in the left posterior temporal area, as well as in both occipital areas. In the brain MRI scan of the PS patient, we observed a $\mathrm{T}_{2}$-high signal in the left hippocampus. These findings suggest that the left temporal and left occipital areas of our patient were irritative and may have elicited the T2-high signal on the brain MRI. Thus, the high $\mathrm{T}_{2}$ signal in the left hippocampus of our patient may not have been an incidental lesion, but instead may be related to the underlying electroclinical diagnosis of PS. The irritative zone of the interictal spikes was clearly related to the ictal onset zone or epileptogenic zone ${ }^{8)}$. Current source analysis including LORETA is one of the most precise techniques for mapping the irritative zone ${ }^{14)}$. The left hippocampus may have been associated with the ictal onset zone or epileptogenic zone in this patient; thus, we hypothesized that the $\mathrm{T}_{2}$ signal change in the left hippocampus was secondary to the vasogenic or cytotoxic edema at the seizure focus. This could be significant because an abnormal $\mathrm{T}_{2}$ signal in the hippocampus may represent an acute stage of hippocampal injury that will progress to hippocampal sclerosis ${ }^{7}$. Although hippocampal pathology in PS has not been reported, prolonged autonomic seizures involving the temporal lobe and accompanied by hypoxemia and/or hypercarbia may have caused hippocampal injury in our patient.

However, it can be argued that the present case is not PS, but symp- tomatic epilepsy with hippocampal pathology because autonomic symptoms, which are well-known characteristics of PS, are common in infants and younger children with temporal lobe epilepsy ${ }^{1}$. However, symptomatic epilepsy in the hippocampus can be ruled out based on the following observations: first, the clinical course of the patient was benign (seizure free over 16 months with low dose oxcarbazepine), and the development status of the patient was normal, which is not characteristic of symptomatic epilepsy. Second, all of the seizures were similar and exhibited the typical PS presentation with autonomic manifestations and a duration longer than 30 minutes. It is well established that PS seizures are typically long ${ }^{3)}$. Third, the EEG findings were not associated with the particular focus expected from the patient's MRI abnormalities. These EEG findings would not be expected in a case of symptomatic epilepsy involving the hippocampus. For these reasons, we diagnosed the present case as PS.

A meaningful attempt was made to determine whether the $T_{2}$ signal changes in the hippocampus of this patient were related to the electroclinical diagnosis of PS or not. Based on the current source distribution of the interictal spikes, we hypothesized that the high $\mathrm{T}_{2}$ signal in the left hippocampus of our patient may not have been an incidental lesion, but instead may be related to the underlying electroclinical diagnosis of PS, particularly seizure. The hypothesis made us to treat immediately with antiepileptic drug in this patient. However, long-term observation and serial follow-up brain MRI may be needed, to confirm the clinical significance of the $T_{2}$ signal change in the hippocampus in this patient.

\section{References}

1. Ferrie CD, Caraballo R, Covanis A, Demirbilek V, Dervent A, Fejerman $\mathrm{N}$, et al. Autonomic status epilepticus in Panayiotopoulos syndrome and other childhood and adult epilepsies: a consensus view. Epilepsia 2007;48: 1165-72.

2. Ferrie C, Caraballo R, Covanis A, Demirbilek V, Dervent A, Kivity S, et al. Panayiotopoulos syndrome: a consensus view. Dev Med Child Neurol 2006;48:236-40.

3. Parisi P, Villa MP, Pelliccia A, Rollo VC, Chiarelli F, Verrotti A. Panayiotopoulos syndrome: diagnosis and management. Neurol Sci 2007;28:72-9.

4. Yalçin AD, Toydemir HE, Celebi LG, Forta H. Panayiotopoulos syndrome with coincidental brain lesions. Epileptic Disord 2009;11:270-6.

5. Cox JE, Mathews VP, Santos CC, Elster AD. Seizure-induced transient hippocampal abnormalities on MR: correlation with positron emission tomography and electroencephalography. AJNR Am J Neuroradiol 1995; 16:1736-8.

6. Kim JA, Chung JI, Yoon PH, Kim DI, Chung TS, Kim EJ, et al. Transient MR signal changes in patients with generalized tonicoclonic seizure or status epilepticus: periictal diffusion-weighted imaging. AJNR Am J Neuroradiol 2001;22:1149-60.

7. Jackson GD, Chambers BR, Berkovic SF. Hippocampal sclerosis: development in adult life. Dev Neurosci 1999;21:207-14. 
8. Engel J, Pedley TA. What is epilepsy? In: Engel J, Pedley TA, Aicardi J, Dichter MA, Moshé S, Perucca E, et al., editors. Epilepsy: a comprehensive text book. 2nd ed. Philadelphia; Lippincott Williams \& Wilkins, 2008:15.

9. Panayiotopoulos CP. Benign nocturnal childhood occipital epilepsy: a new syndrome with nocturnal seizures, tonic deviation of the eyes, and vomiting. J Child Neurol 1989;4:43-9.

10. Leal AJ, Nunes S, Dias AI, Vieira JP, Moreira A, Calado E. Analysis of the generators of epileptic activity in early-onset childhood benign occipital lobe epilepsy. Clin Neurophysiol 2007;118:1341-7.

11. Leal AJ, Nunes S, Martins A, Secca MF, Jordão C. Brain mapping of epileptic activity in a case of idiopathic occipital lobe epilepsy (Panayiotopoulos syndrome). Epilepsia 2007;48:1179-83.

12. Sugita K, Kato Y, Sugita K, Kato M, Tanaka Y. Magnetoencephalographic analysis in children with Panayiotopoulos syndrome. J Child Neurol 2005;20:616-8.

13. Specchio N, Trivisano M, Claps D, Battaglia D, Fusco L, Vigevano F. Documentation of autonomic seizures and autonomic status epilepticus with ictal EEG in Panayiotopoulos syndrome. Epilepsy Behav 2010;19: 383-93.

14. Lüders HO, Najm I, Nair D, Widdess-Walsh P, Bingman W. The epileptogenic zone: general principles. Epileptic Disord 2006;8 Suppl 2:S1-9. 